\title{
CORRECT USE OF COLOR FOR CULTURAL HERITAGE DOCUMENTATION
}

\author{
A. Molada-Tebar, Á. Marqués-Mateu, J.L. Lerma* \\ Photogrammetry \& Laser Scanning Research Group (GiFLE), Department of Cartographic Engineering, Geodesy, and \\ Photogrammetry, Universitat Politècnica de València. 46022 Valencia, Spain. - admote@ doctor.upv.es, (amarques, \\ jllerma)@cgf.upv.es
}

\section{Commission II, WG II/8}

KEYWORDS: Cultural heritage, rock art, colorimetry, CIE color spaces, camera characterization

\begin{abstract}
:
Accurate color recording is a fundamental feature for proper cultural heritage documentation, cataloging and preservation. However, the methodology used in most cases limits the results since it is based either on perceptual procedures or on the application of digital enhancement techniques only. The objective of this study is to apply a rigorous procedure for the characterization of cameras based on a second-order polynomial model. Trichromatic digital cameras capture color information in the well-known RGB format. Nevertheless, the signal generated by the digital camera is device dependent. By means of the characterization, we establish the relationship between device-dependent RGB values and the tristimulus coordinates defined by the CIE standard colorimetric observer. Once the camera is characterized, users obtain output images in the sRGB space that is independent of the sensor of the camera. We applied the methodology on a set of pictures targeting Levantine rock art motifs in Cova Civil (Castellón, Spain) which is considered part of a UNESCO World Heritage Site. We used raw image files, with different exposure conditions, with raw RGB values captured by the sensor. The outcomes obtained are satisfactory and very promising for proper color documentation in cultural heritage documentation.
\end{abstract}

\section{INTRODUCTION}

The integrity of cultural and archaeological heritage is continuously under threat. In recent years, there has been an increase in actions that endanger historical heritage sites by human or natural degradation. Its conservation and maintenance become therefore a priority for professionals in the sector, and it is thus increasingly necessary to collect exhaustive and accurate information to preserve heritage in the future (FernándezLozano et al., 2017).

Moreover, archaeological documentation is a complex process. Nowadays, it is becoming more frequent to combine classical (manual documentation techniques based on perceptual procedures) with rigorous procedures, supported by color measurements and photographs (Cassen et al., 2014; Robert et al., 2016; Rogerio-Candelera, 2015).

The technical measurement and specification of color is a key aspect for proper archaeological documentation (Iturbe et al., 2018; Korytkowski and Olejnik-Krugly, 2017; Ruiz and Pereira, 2014). The correct color definition allows a more comprehensive graphic definition, a better understanding of the scene, and provides relevant technical information especially for research and preservation tasks.

Although techniques have developed remarkably in recent decades, there are still open issues with regard to the correct determination of color. Often, the methodology used for the definition of color in heritage documentation is based on subjective observations, usually by means of color chip collections such as the Munsell charts.
The drawback with this approach is that color vision depends on the observer and therefore it is ultimately a matter of perception. The technical limitations derived from the treatment of color based solely on visual observations are evident.

It is therefore essential to establish a working scheme based on modern methods and digital instrumentation, with a high degree of automation and minimal human intervention. It is important to note, however, that modern technological methods are not exempt from practical or application specific issues.

For instance, contact color measurements in rock art paintings by colorimeters or spectrophotometers are not allowed, in order to avoid any damage to the pigment. On the other hand, the use of digital imaging techniques simplifies the documentation and conservation workflow in cultural heritage tasks, as well as the generation of accurate 3D models.

The problem with the digital approach is that the RGB signals generated by digital cameras are not colorimetrically sound. The output RGB values are said to be device dependent, they do not directly correspond to the device independent tristimulus values based on the Commission Internationale de l'Éclairage (CIE) standard colorimetric observer (CIE, 2004); and do not satisfy the Luther-Yves condition either. Therefore, a digital camera cannot be used a priori for rigorous color determination without any color correction procedure. This was the main motivation to define a rigorous color management workflow as reported in this paper.

A common approach for precise color reproduction based on digital images is based on the use of ICC color profiles or camera profiles (Korytkowski and Olejnik-Krugly, 2017; Ruiz

\footnotetext{
Corresponding author
} 
and Pereira, 2014). Although the use of ICC color profiles offers good results, it is a closed process that requires the use of specific software.

An alternative workflow for correct color registration is the most generic digital camera characterization. Although different mathematical and statistical models such as look-up interpolation tables, artificial neural networks or principal component analysis are used for camera characterization, the most widely used model is the polynomial regression with least squares fitting with different degrees of variation (Hong et al., 2001; Westland et al., 2012). The goal of the camera characterization procedure is to obtain correct colorimetric values from the RGB information registered by the camera sensor through the mathematical relationships between the RGB device dependent color space and the CIE XYZ device independent color space.

Although this methodology is not as accurate as alternative spot-based approaches using colorimeters, several studies show that the results obtained are accurate enough to be used in cultural heritage documentation as well as in other scientific or industrial applications (Martinez-Verdu et al., 2003; MoladaTebar et al., 2018). Thus, the challenge is that a conventional single lens reflex (SLR) digital camera be used for rigorous color determination provided that the camera would had been properly characterized. As a final result, we obtain sRGB images, in a well-known, physically based color space.

Also, in order to determine the color from digital images, the environmental factors involved in the photographic shot, such as sensor, exposure control, lens, ISO or aperture, must be taken into account, since they directly influence the determination of color. The objective of this work is to characterize a set of images of the same rock art scene under different light exposure conditions and analyze the differences.

\section{EXPERIMENT DESCRIPTION}

\subsection{Instrumentation}

As stated above, there are many advantages of using digital images in heritage documentation. The use of digital pictures reduces subjectivity in interpretation, allows the acquisition of RGB data from complete scenes, and increases the precision in graphic and color definition of objects.

Given the importance of color registration, the first aspect to consider is the camera to be used for RGB image capture. From the available options, the camera used in this study was a single lens reflex (SLR) camera SIGMA SD15. The main difference with respect to other commercial digital cameras is the threelayer Foveon X3® direct image sensor (CMOS).

Almost all digital cameras available today contain monochrome image sensors with a color filter array (CFA) which capture all three primary RGB colors at the same time, usually with a Bayer pattern (Figure 1.a.). In those sensors, each pixel captures one primary color only, and the data for the other two colors are obtained by interpolation (also known as demosaicing). On the contrary, Foveon X3® sensor directly captures all primary RGB colors for every single pixel location without interpolation or any optical filter (Figure 1.b.) which makes it a true trichromatic device (Sigma Corporation, 2010).

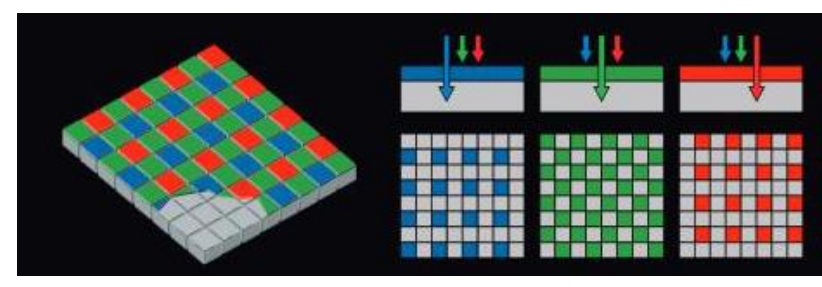

(a)

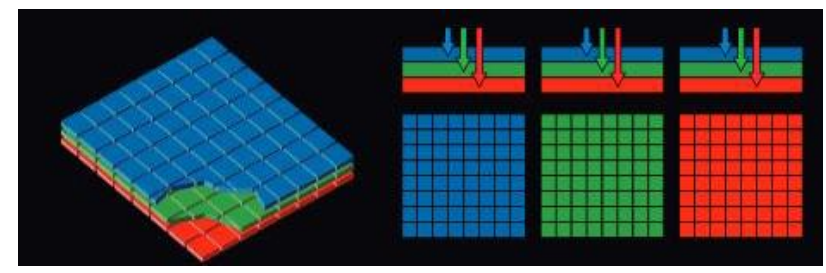

(b)

Figure 1. Incoming radiation and filters used on an image sensor: a) Bayer CFA; b) Foveon X3® (Sigma Corporation,

2010)

All rock art images were taken including a color chart within the scene. The X-rite ColorChecker SG Digital Color Chart was used as color reference. This chart contains 140 colored patches that were used for color characterization (Figure 2).

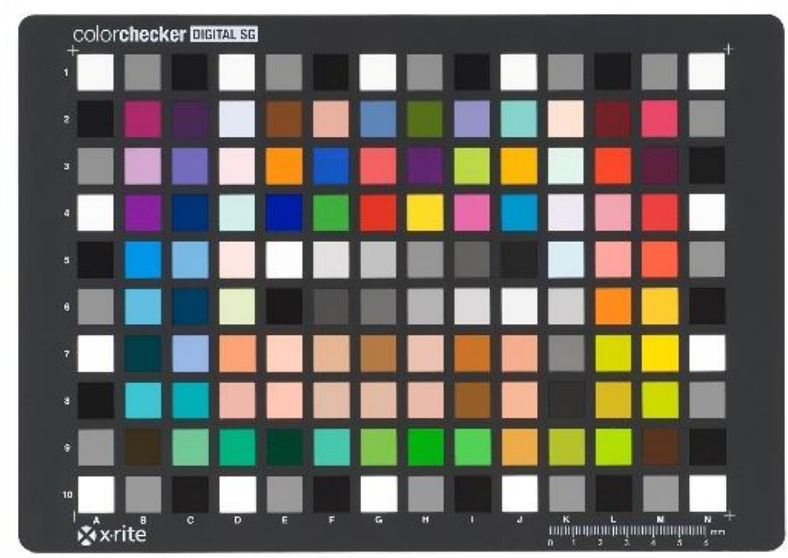

Figure 2. X-rite ColorChecker SG Digital Colour Chart

The CIE XYZ tristimulus coordinates of the color patches present in the color chart are required to conduct the characterization. The manufacturer usually provides these data, however, it is recommended to perform a direct measurement which allows us to control the environmental conditions of the data collection, which in general differ from those of the published chart nominal values.

In our study, we measured the color chart patches under light controlled conditions. The color measurements were obtained with the spectrophotometer Konica Minolta CM-600d, following the CIE recommendations, namely the standard $2^{\circ}$ observer and D65 illuminant (CIE, 2004). 


\subsection{Data processing}

The SIGMA SD15 camera offers raw mode and raw files in the $\mathrm{X} 3 \mathrm{~F}$ data format. Usually, the raw RGB data are transformed by means of a complex sequence of operations to obtain RGB output values. A general workflow for raw image processing is shown in Figure 3, regardles of the camera used for image acquisition. We can differentiate four main phases in the raw image processing pipeline: preprocessing; white balance adjustment, demosaicing (for image sensors with CFA) and color transformation; postprocessing; compressing and storage (Ramanath et al., 2005).

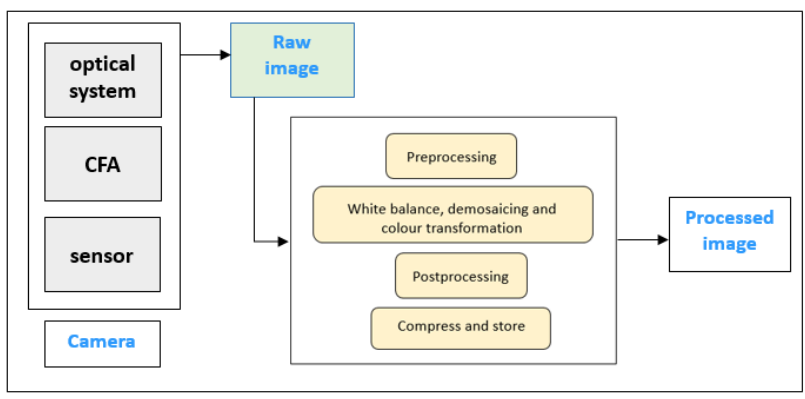

Figure 3. General image processing pipeline

The raw data acquired by the sensor needs to be preprocessed, which includes corrections such as noise reduction; pixel correction; linearization (correction for nonlinear data) and dark current compensation. Before producing a final color image, illuminant correction is applied by means of white balancing. Also, demosaicing (not for Foveon sensor) and color transform is required in order to encode the digital values registered by the sensor. Postprocessing operations, such as color-artifact removal, edge enhancement, and coring are applied for image appearance enhancement. Finally, compression and storage operations are required in order to display or process the final images obtained (Figure 3). It seems clear that, in order to get more consistent color information, it is recommended to use raw image files rather than processed or compressed image files.

The RAW data captured and stored depends on the sensor manufacturer specifications. The X3F RAW file is a binary, complex format specifically suited to Foveon sensors. It is imperative, therefore, to convert the original RAW data into an easily processable format based on numerical arrays. We used the open-source DCRAW decoder to get the original data from raw files as a multidimensional array (Coffin, 2019).

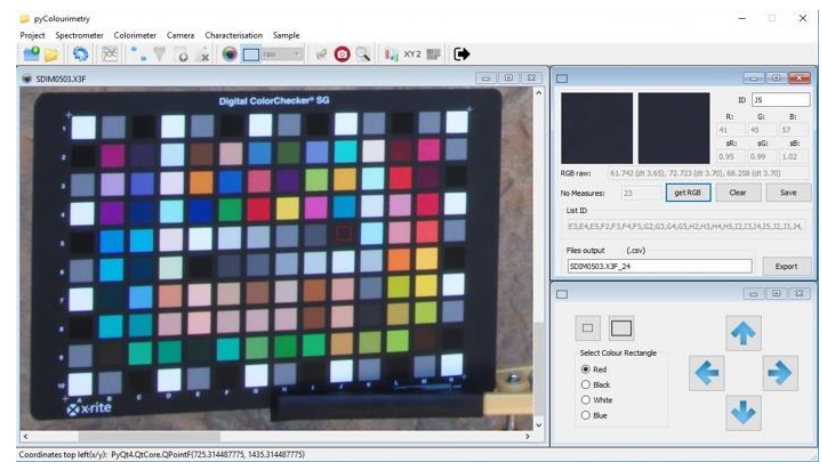

Figure 4. Measuring RGB data with pyColourimetry software
The rigorous processing of colorimetric data requires software packages with specific technical characteristics. In this study, the extraction of RGB raw data from the images (Figure 4), the characterization of the camera and the creation of the output images were accomplished with our own pyColourimetry software (Molada-Tebar et al., 2017), supported with some further analysis in R. This way, we were able to control all the methodological stages comprising the characterization process. Currently, pyColourimetry software is still under evaluation and development phase.

\subsection{Methodology}

The standard way to accurately describe color with a digital cameras is by means of digital image processing after the colorimetric characterisation. In order to conduct the characterisation, we transformed the original data in the device dependent RGB space into the physically based CIE XYZ color space. Results from previous experiences show that good results can be obtained using second order polynomial transformation equations (Balasubramanian, 2003; Westland et al., 2012).

Figure 5 shows the main steps for the image-based characterization workflow proposed.

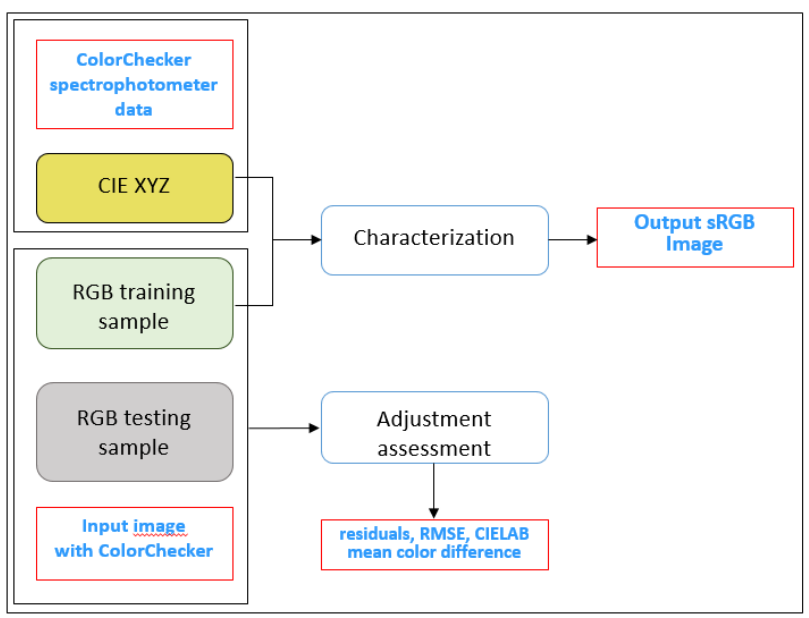

Figure 5. Digital camera characterization workflow

The RGB data captured with the camera are device dependent. Changes in the photographic shot mean changes in the digital values stored on disk files. Thus, a measure of the characterization robustness consists of processing a number of pictures captured under different shot conditions. In order to do so, we collected three pictures to simulate different lights conditions, which led to different and specific corrections for each individual picture. In the end, a set of homogeneous pictures obtained from another set of pictures collected under different environment conditions shows the adaptive nature of the image-based characterization.

In the process, we need several sets of samples with RGB data from the selected color patches. A training sample will be used asa reference for setting the characterization parameter, which ultimately establishes the relationship between the RGB data and the CIE XYZ tristimulus coordinates. Additionally, a testing sample to evaluate the quality of the applied adjustments is required. 
Color accuracy can be measured by computing the CIE76 color difference formula $\Delta \mathrm{E}_{\mathrm{ab}}^{*}$ (CIE, 2004) between the imaging results of the standard target patches and their theoretical color values. In this study, we used the CIE76 color difference standard instead of other most recent formulas such as the CIEDE2000 because the CIE76 fits better to rock art working environments (Molada-Tebar et al., 2018).

Specifically, fieldwork conditions in archaeologymake the use of the CIEDE2000 and similar formulas unsuitable in practical terms. We should note that the reference conditions required for the use of the CIEDE2000 formulae were developed for industrial purposes under laboratory conditions (CIE, 2014; Luo et al., 2001).

Finally, once the transformation equations are determined, we can represent the output characterized image in the sRGB color space.

\section{CASE STUDY}

The data used in the experiment consisted of a set of digital images taken on a rock art site, called Cova Civil, located in Tirig, Castellón (Figure 6). This site is one of the most singular rock art caves of the Mediterranean Basin in the Iberian Peninsula that was included in the UNESCO World Heritage list in 1998 (Unesco, 2019).

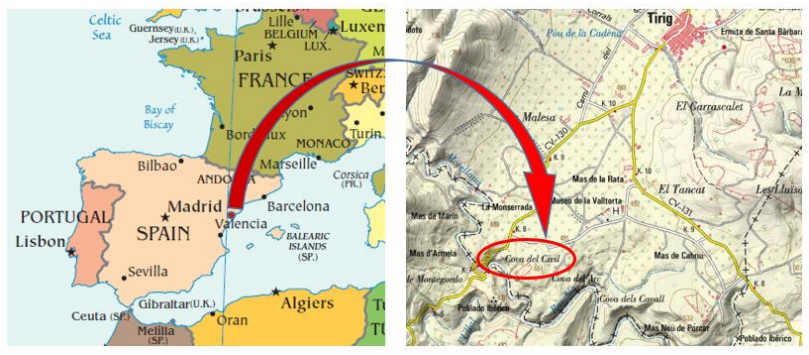

Figure 6. Location map of Cova Civil rock art site

All images correspond to the same scene, taken at the same time, under the same lighting conditions (under the hypothesis of D65 illumination), ISO, aperture and focal (ISO 100, f/22, 20 $\mathrm{mm})$. The only variable photographic parameter wasexposure (Table 1). Three pictures were selected from the original set: an image with normal exposure (1/5 s), another with overexposure $(0.6 \mathrm{~s})$, and finally one picture with underexposure $(1 / 25 \mathrm{~s})$. The images used are shown in Figure 7.a., 8.a. and 9.a. respectively.

From the raw RGB data of the color patches and their theoretical CIE XYZ values, we obtained characterization parameters by means of second-order polynomials, which establish the relationship between the device dependent RGB space and the CIE XYZ space defined by the CIE standard colorimetric observer.

In order to assess the results obtained after characterisation, we used the CIE XYZ residuals root mean square error (RMSE) and color differences between computed and theoretical data. The results obtained for each of the images used are shown in Table 1.

\begin{tabular}{|c|c|c|c|c|c|}
\hline \multirow{2}{*}{ Image } & \multirow{2}{*}{$\begin{array}{c}\text { Exposure } \\
(\mathrm{s})\end{array}$} & \multicolumn{3}{|c|}{ RMSE CIE XYZ } & \multirow{2}{*}{$\Delta \mathrm{E}_{2 \mathrm{a}}^{\mathrm{m}}$} \\
\cline { 3 - 5 } & & $\mathrm{X}$ & $\mathrm{Y}$ & $\mathrm{Z}$ & \\
\hline SDIM0498 & $1 / 5$ & 0.7040 & 0.6368 & 0.8551 & 2.1968 \\
\hline SDIM0501 & 0.6 & 1.0470 & 1.5624 & 0.9257 & 4.9267 \\
\hline SDIM0505 & $1 / 25$ & 0.7545 & 0.5896 & 0.7104 & 2.4333 \\
\hline
\end{tabular}

Table 1. Characterization results. RMSE and $\triangle E_{a t h}^{\infty}$ CIELAB values.

In this study, as explained in section 2.3, we used the CIE76 color difference formula $\Delta \mathrm{E}_{\mathrm{ab}}^{*}$ (CIE, 2004). We used the CIELAB coordinates instead of the CIE XYZ tristimulus values because of the uniformity characteristics of the former. The CIELAB space also provides a rigorous formula to compute color difference metrics.

Finally, once the camera was characterized, we obtained output images in the sRGB space, which is known to be device independent and allows right color rendering (Figure. 7.b., 8.b., 9.b.)

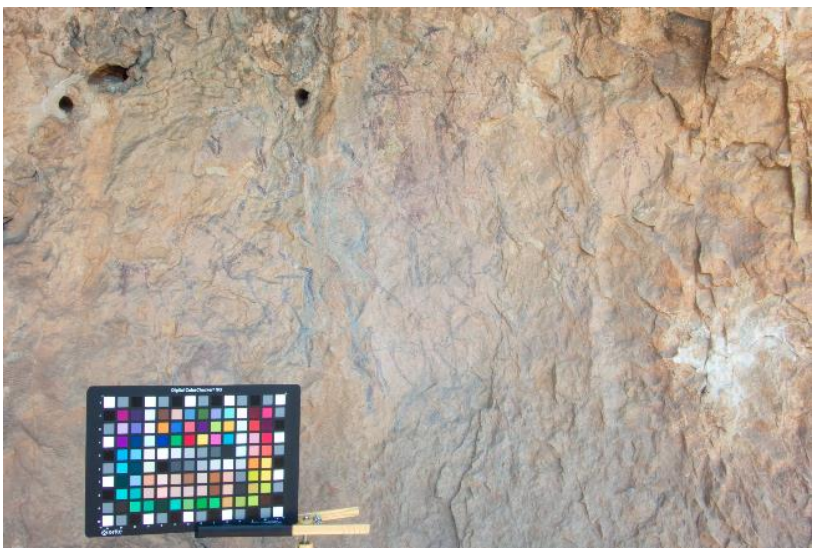

(a)

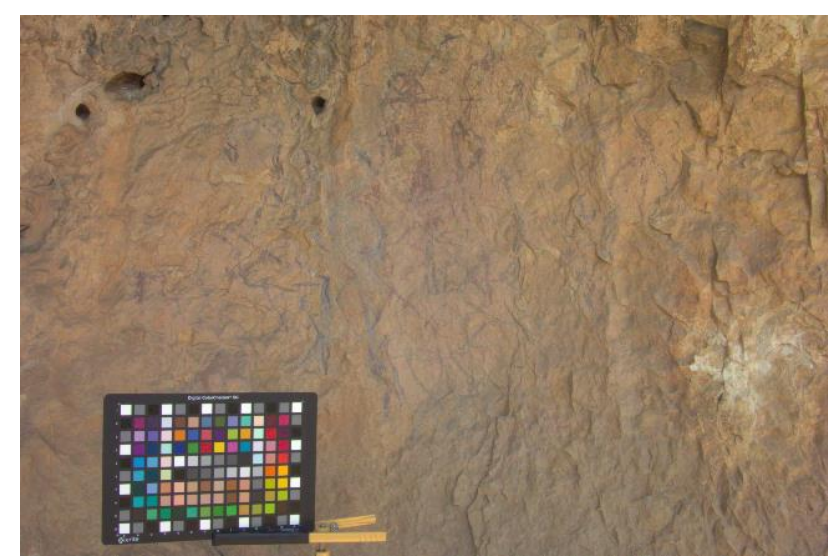

(b)

Figure 7. Image SDIM0498.X3F: a) Original RGB image; b) sRGB output image 


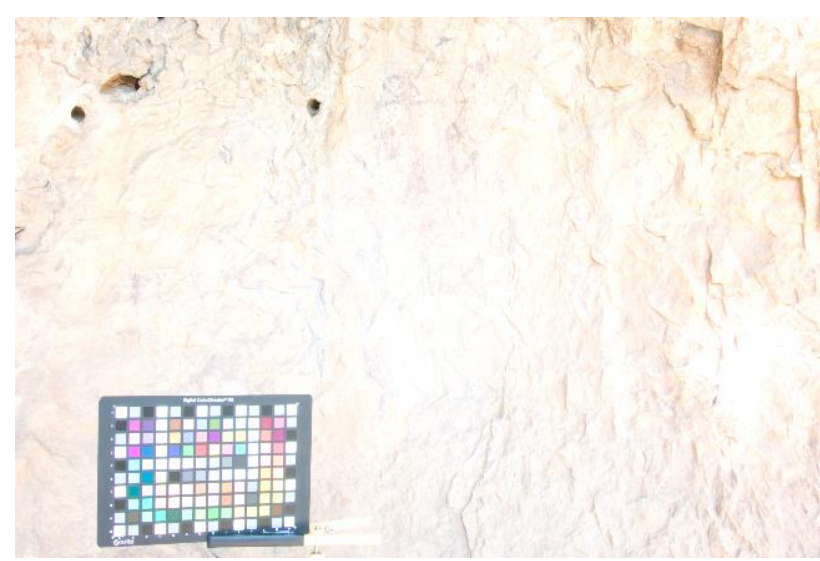

(a)

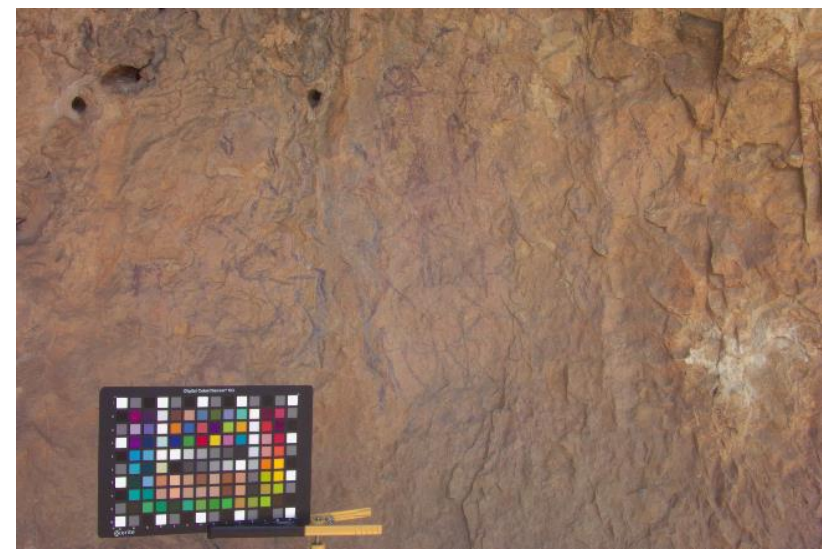

(b)

Figure 8. Image SDIM0501.X3F: a) Original RGB image; b) sRGB output image

\section{DISCUSSION}

Digital description by photographic images in heritage documentation is a suitable technique due to characteristics such as its low cost, non-destructive data acquisition, ease and speed of collection, and the diversity of software for the postprocessing stages. However, in many studies, the color definition of the archaeological artifacts is limited by exposure compensation, optical image correction, denoising, sharpening, color balance or enhancement techniques (Apollonio et al., 2014; Palomar-Vazquez et al., 2017).

Considering the RMSE metrics obtained in this study, it is observed that the adjustment with second-order polynomials is adequate. We obtained lower values except for the image with overexposure (Table 1). Regarding residual color differences, values below three CIELAB units indicated satisfactory results, since those color differences are hardly perceptible by the human eye (Mahy et al., 1994).

It should be noted that we always found differences below three CIELAB units except for the overexposed picture (4.9). This is an expected outcome since color perception depends on light, and therefore extreme lighting conditions do modify fundamental colorimetric factors such as the lightness and even the color appearance of the object (Figure 8.b).

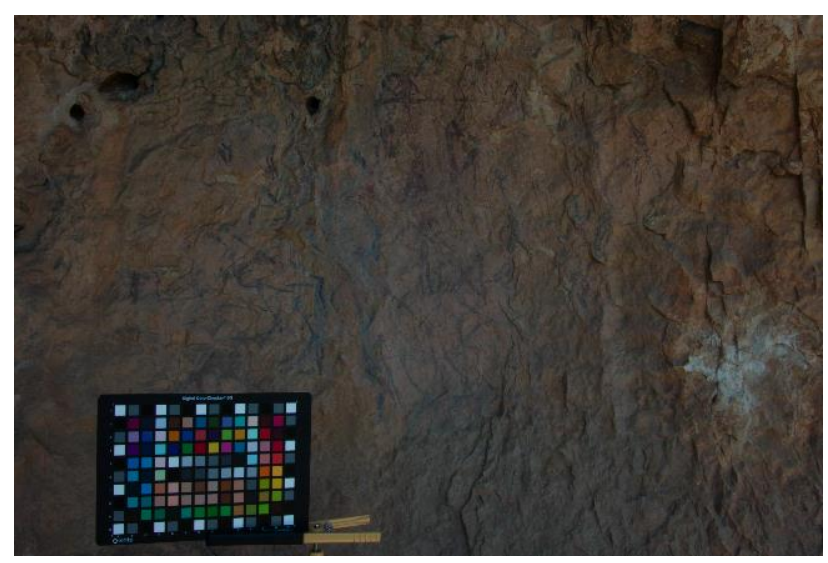

(a)

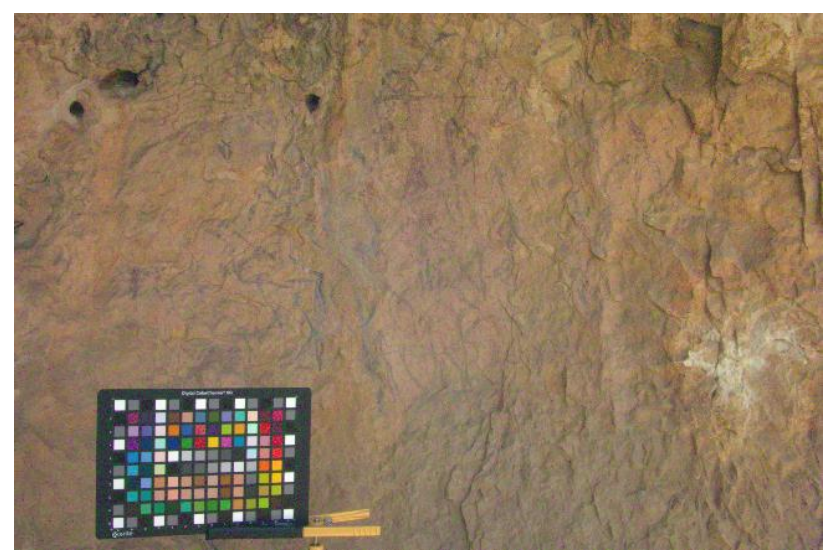

(b)

Figure 9. Image SDIM0505.X3F: a) Original RGB image; b) sRGB output image

The best results were found for the SDIM0498 and SDIM0505 images, with CIE76 differences under three CIELAB units (2.2 and 2.4 respectively). The output sRGB characterized imagesoffer similar results, and it is not possible to perceive the difference between both in terms of color differences (Compare Figure 7.b. with Figure 9.b).

In view of these outcomes, the results of the experiment are satisfactory and very promising for proper color documentation in rock art studies. The rigorous procedure proposed for digital cameras characterization allows users to achieve accurate color recording and rendering.

This workflow is based on objective methods that are independent of the observer experience. It combines the direct method, based on colorimetric measurements, and the indirect method, using digital images with RGB information (MartínezVerdú et al., 2003; Westland et al., 2012).

\section{CONCLUSIONS}

Definitely, the correct description of color is a fundamental task in heritage documentation and preservation. We need to definethe color and its changes over time in a rigorous fashion which still remains a complex issue for some users. 
In this paper it can be seen that colorimetry allows the characterization of color in a non-subjective way by means of the well-known CIE tristimulus values approach. Through the characterization process, we can determine the numerical relationships between the RGB device dependent color space (for each setup) and the standard CIE XYZ color space.

The methodology proposed is a reliable and low-cost solution for correct color specification and communication. Characterization relies on the well-defined second order polynomial model, which is adequate and takes into account the most important technical colorimetric aspects. In addition, it allows the registration of color as close as possible to reality, obtaining colorimetric information of complete scenes; and the comparison of images acquired in different exposure conditions by means of a common sRGB color space.

Archaeological documentation greatly benefits from this colorimetric workflow. With this SLR camera-based characterization approach, users can collect rigorous color information regardless of the device used in the data acquisition, relying on digital images including a colorchart and colorimetric measurements only. Furthermore, this process is not restrictive and can be used in combination with other common techniques used for the study and conservation of archaeological sites such as laser scanning or photogrammetric techniques.

\section{ACKNOWLEDGMENTS}

This research is partly funded by the Research and Development Aid Program PAID-01-16 of the Universitat Politècnica de València, through FPI-UPV-2016 Sub 1 grant. The authors gratefully acknowledge the support from the Spanish Ministerio de Economía y Competitividad to the project HAR2014-59873-R. The authors also would like also to acknowledge the comments from the colleagues at the Photogrammetry \& Laser Scanning Research Group (GiFLE).

\section{REFERENCES}

Apollonio, F.I., Ballabeni, M., Gaiani, M., 2014. Color enhanced pipelines for reality-based 3D modeling of on site medium sized archeological artifacts. Virtual Archaeology Review, 5 (10), pp. 59-76.

Balasubramanian, R., 2003. Device characterization. Digital Color Imaging Handbook, Chapter 5. CRC Press.

Cassen, S., Lescop, L., Grimaud, V., Robin, G., 2014. Complementarity of acquisition techniques for the documentation of Neolithic engravings: Lasergrammetric and photographic recording in Gavrinis passage tomb (Brittany, France). Journal of Archaeological Science, 45, pp. 126-140.

CIE. 2004. CIE 015:2004. Colorimetry. Commission Internationale de l'Eclairage.

CIE, 2014. ISO/CIE 11664-6:2014(E). Colorimetry-Part 6: CIEDE2000 Colour-Difference Formula. Commission Internationale de l'Éclairage.

Coffin, D., 2003. Decoding raw digital photos in Linux. URL https://www.cybercom.net/ dcoffin/dcraw/ (1 Feb 2019)
Fernández-Lozano, J., Gutiérrez-Alonso, G., Ruiz-Tejada, M.Á., Criado-Valdés, M., 2017. 3D digital documentation and image enhancement integration into schematic rock art analysis and preservation: The Castrocontrigo Neolithic rock art (NW Spain). Journal of Cultural Heritage, 26, pp. 160-166.

Hong, G., Luo, M.R., Rhodes, P.A., 2001. A study of digital camera colorimetric characterization based on polynomial modeling. Color Res. Appl. 26(1), pp. 76-84.

Iturbe, A., Cachero, R., Cañal, D., Martos A. 2018. Virtual digitization of caves with parietal Paleolithic art from Bizkaia. Scientic analysis and dissemination through new visualization techniques. Virtual Archaeology Review, 9 (18), pp. 57-65.

Korytkowski, P., Olejnik-Krugly, A., 2016. Precise capture of colors in cultural heritage digitization. Color Res. \& Appl. 42 (3), pp. 333-336.

Luo, M.R., Cui, G., Rigg, B., 2001. The development of the CIE 2000 colour-difference formula: CIEDE2000. Color Res. Appl. 26(5), pp. 340-350.

Mahy, M., Van Eycken, L., Oosterlinck, A., 1994. Evaluation of Uniform Color Spaces Developed after the Adoption of CIELAB and CIELUV. Color Res. \& Appl. 19 (2), pp. 105121.

Martínez-Vedú, F., Pujol, J., Capilla, P., 2003. Characterization of a Digital Camera as an Absolute Tristimulus. Proceedings of SPIE - The International Society for Optical Engineering, 47, pp. 279-295.

Molada-Tebar, A., Lerma, J.L., Marqués-Mateu, A, 2018. Camera characterization for improving color archaeological documentation. Color Res. \& Appl., 43 (1), pp. 47-57.

Molada-Tebar, A., Lerma, J.L., Marqués-Mateu, A, 2017. Software Development for Colourimetric and Spectral Data Processing: Pycolourimetry. Proceedings of 1st Congress in Geomatics Engineering, pp. 48-53.

Palomar-Vazquez, J., Baselga, S., Viñals-Blasco, M.-J., GarcíaSales, C., Sancho-Espinós, I., 2017. Application of a combination of digital image processing and $3 \mathrm{D}$ visualization of graffiti in heritage conservation. Journal of Archaeological Science: Reports, 12, pp. 32-42.

Ramanath, R., Snyder, W.E., Yoo, Y., Drew, M.S., 2005. Color image processing pipeline. IEEE Signal Processing Magazine, 22 (1), pp. 34-43.

Robert, E., Petrognani, S., Lesvignes, E., 2016. Applications of digital photography in the study of Paleolithic cave art. Journal of Archaeological Science: Reports, 10, pp. 847-858.

Rogerio-Candelera, M.Á., 2015. Digital image analysis based study, recording, and protection of painted rock art. Some Iberian experiences. Digital Applications in Archaeology and Cultural Heritage, 2 (2-3), pp. 68-78.

Ruiz, J.F., Pereira, J., 2014. The colours of rock art. Analysis of colour recording and communication systems in rock art research. Journal of Archaeological Science, 50 (1), pp. 338349. 
Sigma Corporation, 2010. The SIGMA SD15 digital single lens reflex camera with Foveon ${ }^{\circledR}$ full color sensor. Sigma corporation.

Unesco. Rock Art of the Mediterranean Basin on the Iberian Peninsula. URL http://whc.unesco.org/en/list/874 (1 Feb 2019).

Westland, S., Ripamonti, C., Cheung, V., 2012. Computational Colour Science using MATLAB®. John Wiley \& Sons, Ltd, Chichester, UK. 